Article

\title{
Development and Application of a Planning Support System for Regional Spatial Functional Zoning Based on GIS
}

\author{
Xiaorui Zhang ${ }^{1,2, *}$, Qian Hua ${ }^{1}$ and Linya Zhang ${ }^{1}$ \\ 1 Department of Urban Planning, Hefei University of Technology, Hefei 230009, China; \\ clhhfut@126.com (Q.H.); zxrl@sina.com (L.Z.) \\ 2 Laboratory of Digital Human Habitat Studies, Hefei University of Technology, Hefei 230009, China \\ * Correspondence: rgdhf@hfut.edu.cn; Tel.: +86-551-6468-3152
}

Academic Editor: Vincenzo Torretta

Received: 16 July 2016; Accepted: 2 September 2016; Published: 7 September 2016

\begin{abstract}
Regional spatial functional zoning in China is a large-scale strategic planning and requires very advanced planning decision support technology. With respect to spatial functional zoning, the planning support system (PSS) is introduced as one of the most popular computer aided planning systems at the present time in this paper, and is further explained with the example of the latest regional main functional area (RMFA) planning in China. On the Visual Studio.NET visualization development platform, the planning decision model, planning decision method, and GIS are organically integrated and customized by using the component-type secondary development technology of the ArcGIS Engine with the development language VB.NET. Hence, a highly efficient and flexible planning support system of regional main functional areas (RMFA-PSS) is established. It was adopted in the research of the Beijing-Tianjin area in China and accordingly two types of main functional area planning are acquired. The RMFA-PSS offers an efficient and flexible decision support with regard to RMFA planning. The research can provide a reference for further PSS development and applications related to other types of spatial functional zoning.
\end{abstract}

Keywords: planning support system (PSS); spatial functional zoning; GIS; regional main functional areas (RMFA); Beijing-Tianjin area in China

\section{Introduction}

In the pattern of national development strategy, each regional space assumes a certain function. Regional spatial functional zoning provides scientific evidence in order to establish a regional development layout with clear function, reasonable division of labor, and complementary advantages. Moreover, it can play an important role in improving spatial allocation efficiency and optimizing spatial structure of the regional socio-economic [1]. As the latest frontier domain in planning subject development, the regional spatial functional zoning has been considered as the strategic starting point for national, regional, and local planning [2]. This can be seen in the European transformation from spatial planning to spatial regulation, in the American transformation from "simple zoning" to the "smart growth", in the Japanese and Korean transition from land comprehensive improvement to sustainable development planning, and also China's transformation from comprehensive natural division and ecological functional zoning to the latest main functional zoning. Compared with small-scale city planning and construction, regional spatial function zoning is macro-planning on a larger scale. It involves all aspects of the regional economy, society, and the natural environment [3], and is more complicated in the planning index system, planning model, data processing, etc. In a word, it requires more scientific planning, technological support, especially the computer aided planning 
system, an integrated planning model, method, result analysis, and output. Thereby, planning efficiency and precision is improved.

Planning Support System (PSS) is currently one of the most prevalent computer aided planning systems [4]. Harris (1989) first put forward the concept of PSS [5]. He reckoned that in combination with spatial information technology and planning methods and steps, PSS can provide decision support for different stages in the planning process. Once this concept was proposed, it received considerable attention and concern, and has become a significant research area and the main development direction in the computer aided planning system [6]. Essentially, PSS is affiliated within the category of the earlier developed decision support system (DSS), but it is a special type of DSS. It assists planners with their specific planning task, particularly suitable for decision support in a variety of professional plannings.

In developed countries, PSS has the function of a decision support system and offers various tools, models, methods, and especially some specialized technologies in the planning area. The aim of PSS is to solve the problems in semi-structured spatial decision, such as urban growth [7], land utilization [8], land allocation and consolidation [9], urban land policy formulation [10], sustainable development planning [11], urban form situation assessment [12], urban infrastructure planning and construction [13], and transportation planning [14]. Meanwhile, foreign states have developed some practical PSS, for instance, CUF, WHAT IF?, SLEUTH, INDEX, CommunityViz, CITYgreen, GB-QUEST etc. [15]. In comparison, PSS research in China is still in its infancy, but is developing rapidly. The existing research mainly covers: studying the urban master planning layout in the light of foreign PSS [16]; introducing and analyzing basic issues in PSS including concepts, theories, and methods $[17,18]$; developing and applying PSS to several newly-exploited areas, such as strategic environmental decision assessment systems concerning urbanization [19], land use planning support systems for urban and rural areas [20], planning support systems related to urban growth control [21], and agricultural land optimization systems [22].

However, research on specialized regional spatial functional zoning can rarely be found from the above PSS research and application, whether home or abroad. In other words, research in this area should be initiated and encouraged. Therefore, this paper aims to introduce PSS to the field of spatial functional zoning including its concept and principles and the development and application of PSS in the latest regional main functional area (RMFA) planning in China. The discussion is based on the geographic information system (GIS) which is regarded as the basic technical platform for secondary development via computer programming language. In this way, the planning decision model, planning decision method, and GIS are organically integrated and customized thereby establishing the planning support system for the regional main functional areas (RMFA-PSS) in China. Moreover, it has been applied to the main functional zoning in research of the Beijing-Tianjin area in China. The purpose of this article is to provide a case study and application for the decision support technology in the field of regional spatial functional zoning, which is expected to provide a reference on the theory and technology towards future studies in related areas.

\section{Principles and Methods}

\subsection{PSS Principles}

The planning support system is developed by extending the traditional concept of DSS into planning and is closely connected with GIS technology (Figure 1). PSS, within spatial analysis technology of GIS, can provide auxiliary decision support with various planning issues and in turn, realize integration and linking between spatial analysis, the planning decision model, and the method. The basic PSS principles can be summarized as the following three points. 


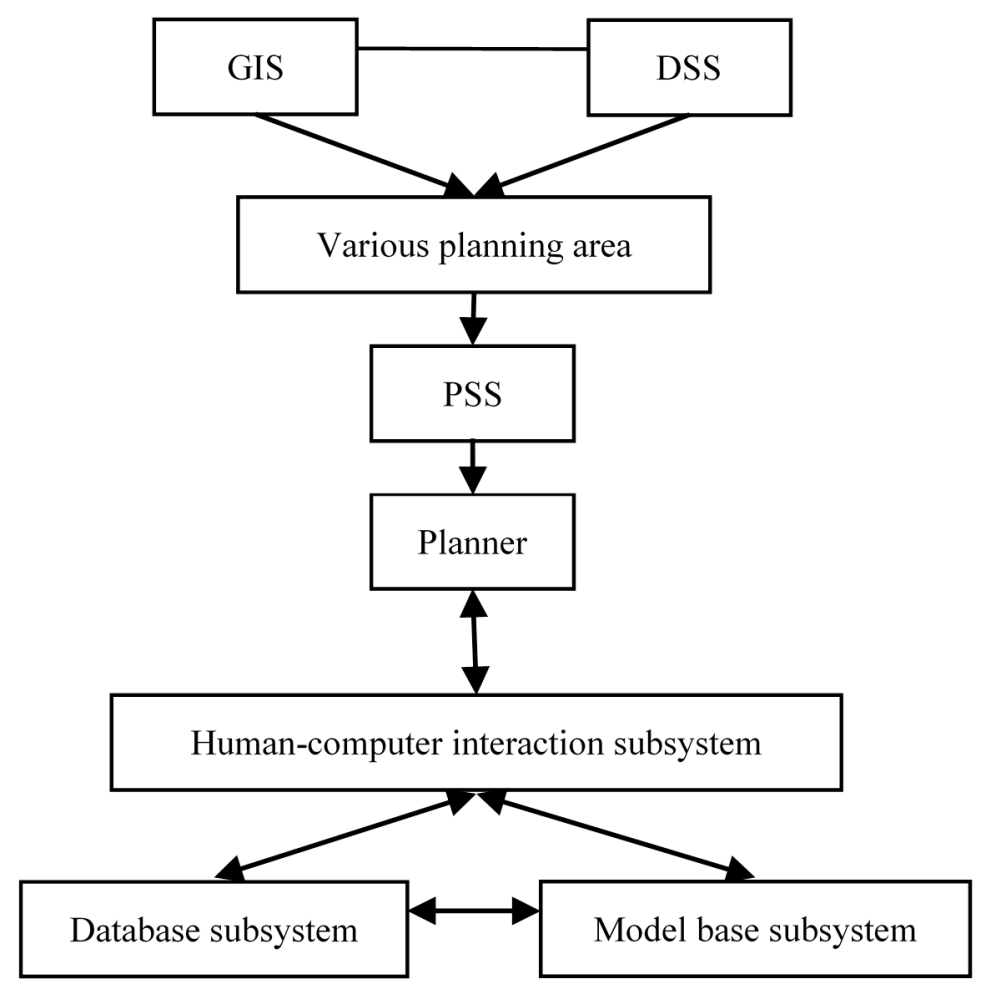

Figure 1. The source and basic structure of the planning support system (PSS).

(1) PSS is a subset of DSS, referring to a decision support system which integrates GIS technology, the planning decision model and various technologies directly related to planning. As a decision-making interactive computer system, it is used to support one or a set of planners in semi-structured or unstructured planning decisions at high efficiency. The comparison of PSS and DSS is shown in Table 1.

(2) The PSS is an auxiliary decision-making tool, offering support to decision making in various planning stages, rather than making decisions by itself. PSS aims to expand the capacity of decision-making of planners, rather than replace them, planners always retain autonomy in decision-making. At the same time, PSS does not directly offer the best solution, but through scenario planning analysis presets several possible strategies and planning rules, and then simulates each option, under planning strategies and rules by PSS, and uses visualization methods such as images and diagrams of the different results communicated to planners. Finally, it depends entirely on the power of planners to choose a scheme and planners will need to choose the most suitable planning scheme from decision results. The PSS internal decision-making process is completely transparent, in which planners can clearly grasp planning strategies and rules; by changing these strategies and rules, PSS will be able to produce different planning results. Hence there will not be PSS without planning strategies and decision making rules. The condition and foundation of PSS development and application is the support from planning strategies and decision-making rules. The essence of the PSS is to provide a technology platform for multi-scheme comparison based on different planning strategies and decision-making rules.

(3) The basic structure of PSS includes the database subsystem, model base subsystem, and human-computer interaction subsystem. The database subsystem mainly copes with comprehensive building concerning different data types. The model base subsystem mainly copes with model building, model algorithm and the integrated specialized model with GIS. The human-computer interactive subsystem mainly deals with the interactive communication between user and system, input and output, as well as the planning decision-making processes and results visualization. The model is the core of PSS. Without the model, PSS could neither 
provide planning decision support function, nor be an authentic PSS. At best, it could be a planning information system. According to the existing PSS, CUF integrates the population prediction model and spatial allocation model, "WHAT IF?" integrates the land suitability evaluation model, the land demand forecasting model and land use allocation model, and SLEUTH results from the cellular automata model. Thus, PSS is "model-driven". There would be no PSS without a planning decision model.

Table 1. Comparison between planning support system (PSS) and decision support system (DSS).

\begin{tabular}{ccc}
\hline Content Comparison & DSS & PSS \\
\hline Data form & Non-spatial data & Spatial data and non-spatial data \\
\hline Data acquisition & Single: statistics & Variety: statistical, digitized scanning, image processing, etc. \\
\hline Decision model & Non-spatial model & Spatial model based, supplemented by non-spatial model \\
\hline Results output & Figures, tables & Graphics, images, tables, etc. \\
\hline Combined with GIS & Generally no & Closely combined with GIS, there would be no PSS without GIS \\
\hline
\end{tabular}

\subsection{RMFA Principles}

Regional main functional areas (RMFA) is the latest regional spatial functional zoning in China. It was launched as a national strategic promotion project in 2006. According to the regional resource-environment carrying capacity, existing development density, and development potential, RMFA planning divides national space into four main functional zones: prohibited developing area (PDA), restricted developing area (RDA), focus developing area (FDA), and optimized developing area (ODA). Not only is this a new ground towards the establishment of an orderly regional development pattern in China, but also a major innovation regarding a national land spatial development system and mechanism [23].

\subsubsection{RMFA Planning Model}

The core of RMFA planning is a set of planning decision-making models and methods, and of course, also a prerequisite of RMFA-PSS development and application. According to relevant research [24], this paper takes the four-force model "carrying capacity-potential-pressure-resistance" as the decision model of RMFA-PSS development.

In the four-force model, the sum of the resource environmental carrying capacity and the economic-social potential constitutes the "total potential" or impetus in regional spatial development, while the sum of environmental stress and ecological resistance constitutes the "total resistance". Referring to the cost-benefit analysis method, the integrated planning index (IPI) is the resultant force of four forces. It can be seen as the rest of the driving force deducting the delaying force of regional spatial development, $I P I=$ (Carrying capacity + Potential $)-($ Pressure + Resistance $)$. From this point of view, the general layout of the RMFA planning strategy is to follow the decision rules that "index system-index normalizing score-index weight—index synthesis". Each of the four forces can correspond to a set of evaluation index system. The absolute value after index normalization and index synthesis is the size of each force. Therefore, the problem of four forces synthesis can be solved by scientifically determining weight, normalizing score, and overlay rule. The specific calculation steps of IPI are as follows.

First is the data normalization. Formula (1) is to calculate the positive index, formula (2) is to calculate the negative index.

$$
\begin{aligned}
& x_{i}=\frac{x-x_{\min }}{x_{\max }-x_{\min }} \\
& x_{i}=\frac{x_{\max }-x}{x_{\max }-x_{\min }}
\end{aligned}
$$


In the formulas (1) and (2), $x_{i}$ is the normalized evaluation index value, $x$ is the original index value, $x_{\max }$ is the maximum value of the original indexes, $x_{\min }$ is the minimum value of the original indexes. Secondly, the size of the four forces should be calculated. The formula is:

$$
F=\sum_{i=1}^{n} w_{i} x_{i}
$$

In the formula (3), $F$ is the magnitude of each force, $x_{i}$ is the normalized evaluation index value of the force, $w_{\mathrm{i}}$ is the corresponding weight of each evaluation index of the force.

Finally, the resultant force of the four forces is calculated, and then the IPI of each spatial unit is obtained. It can specify per spatial unit into the corresponding main functional areas according to the IPI. The formula is:

$$
I P I=\left(W_{a} F_{a}+W_{b} F_{b}\right)-\left(W_{c} F_{c}+W_{d} F_{d}\right)
$$

In the formula (4), $F_{a}, F_{b}, F_{c}, F_{d}$ respectively stand for resource-environmental carrying capacity, economic-social potential, environmental stress and ecological resistance. $W_{a}, W_{b}, W_{c}, W_{d}$ respectively represent the weight of each corresponding force, $W_{a}+W_{b}+W_{c}+W_{d}=1$.

Formulas (1) and (2) can solve the problem of index normalization. Formulas (3) and (4) give the methods of index synthesis, which is also the main decision rule of RMFA planning. Obviously, a large number of index weight calculations are involved in these decision rules. Weight reflects the decision preference and planning strategy of planners, which can be calculated by using a variety of methods such as the commonly used AHP (Analytical Hierarchy Process) method, the sort method or user defined, etc.

According to formula (4), IPI refers to the standardized resultant force against regional spatial development. It is a comprehensive measurement evaluating the condition of regional spatial development. The values of IPI of four major functional zones descend as follows: ODA > FDA $>$ RDA $>$ PDA (Figure 2). According to Figure 2, Point 0 is the demarcation point of developed function areas (ODA and FDA) and protected function areas (RDA and PDA), Point A is the demarcation point of ODA and FDA, and Point $B$ is the demarcation point of RDA and PDA. However, based on specific planning purposes, planners can flexibly set different values. The fixed Point 0 and the flexible Point A and Point B embody the most obvious characteristics of the model, illustrating the organic unity of rigid constraint and flexible control. Further details about the planning model can be seen in the Reference [24].

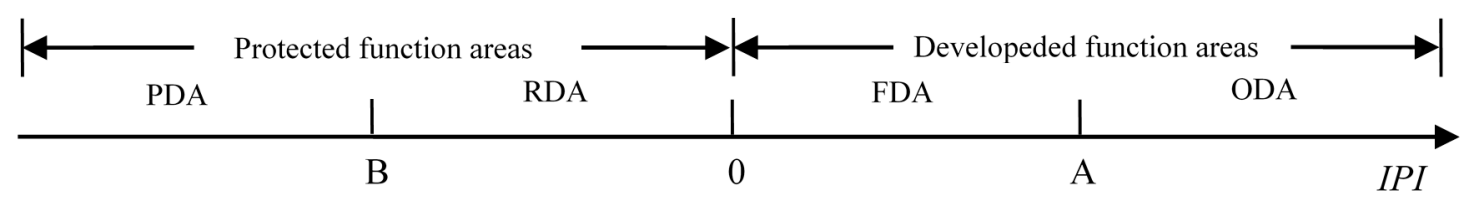

Figure 2. The order of division of major functional areas in accordance with IPI.

\subsubsection{RMFA Planning Methods}

Planning decision method is a series of comprehensive technological approaches pursuing a solution method based on the planning decision model. Under the guidance of the RMFA planning model and the multi-criteria decision method, the RMFA planning decision process can be summarized as: planning index system building, index standardized score, index weight calculating, index synthesizing and integrated planning index. From the perspective of decision theory analysis, RMFA planning is a typical multi-criteria decision-making process on the basis of a comprehensive evaluation index system, within which the score, the weight and the decision rule constitute the three major elements in the multi-criteria decision-making. When operating, each set of evaluation indexes should 
be selected with regard to the prescribed content in accordance with each force. Generally, the process of the RMFA planning decision method can be summed up as in Figure 3.

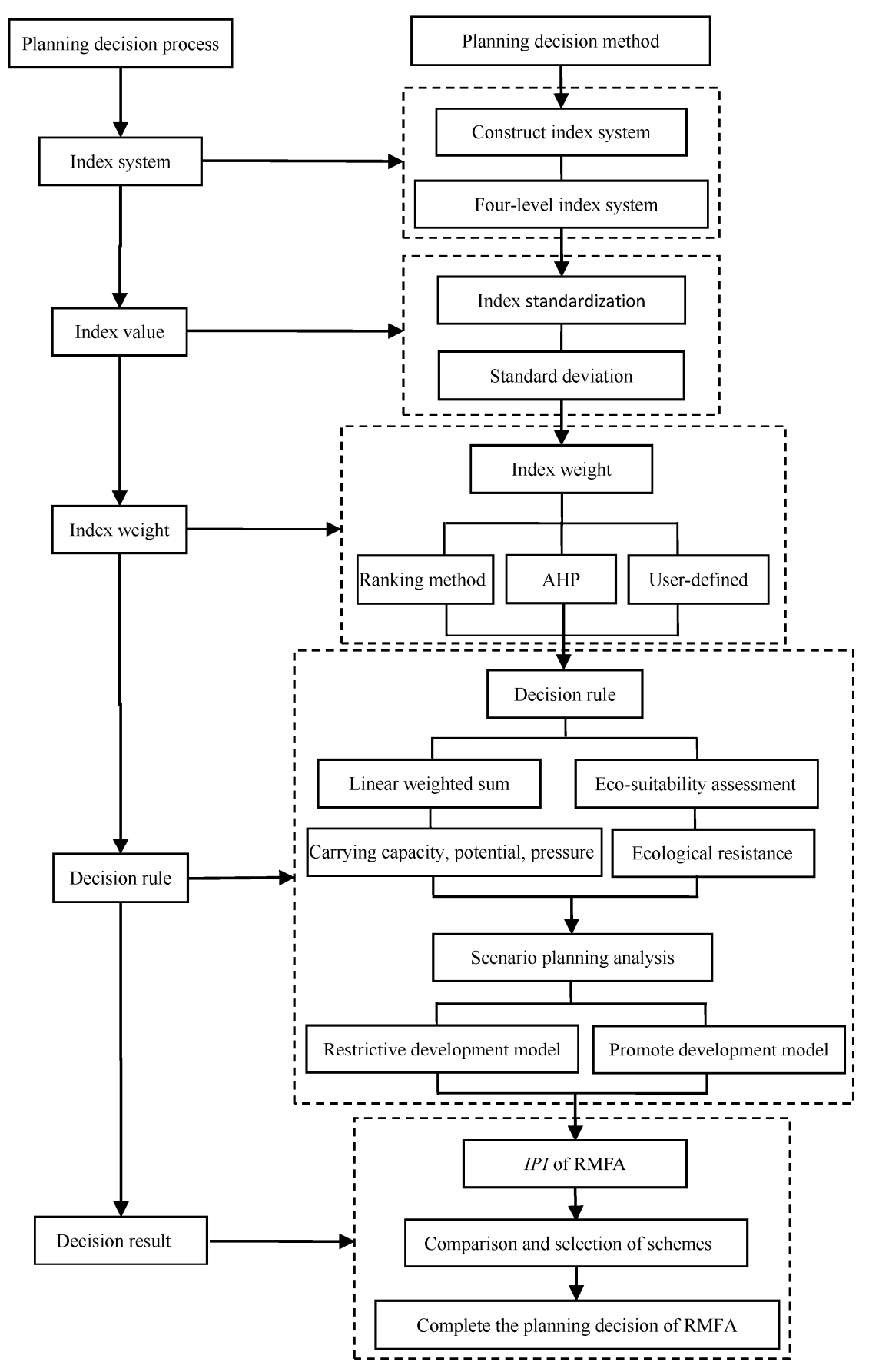

Figure 3. The process of the RMFA planning decision method.

\section{Development of RMFA-PSS}

\subsection{Development Platform}

RMFA-PSS works on the basis of the secondary development mode in GIS components. This mode combines GIS technology with component technology, which has now become the mainstream in GIS development. Its basic idea is that with classification of various functional GIS models into different types of control, each control completes its corresponding function, while between either 
GIS or non-GIS controls, the integration can be realized through visible software development tools [25]. In this way, the GIS application system will meet the user's specific need. Such secondary component development mode provides new tools for the application of the new GIS generation with various features and benefits, including integrated flexibility, low cost, development convenience, utility convenience, easy generalization, interface visualization, etc. Specifically, on the Visual Studio.NET (.NET) visualization development platform and by using the ArcGIS Engine (AE) technology and VB.NET language, RMFA-PSS has realized the comprehensive integration of decision model, decision-making method, as well as common GIS and other non-GIS functions. The RFMA-PSS development platform can be concluded as in Figure 4.

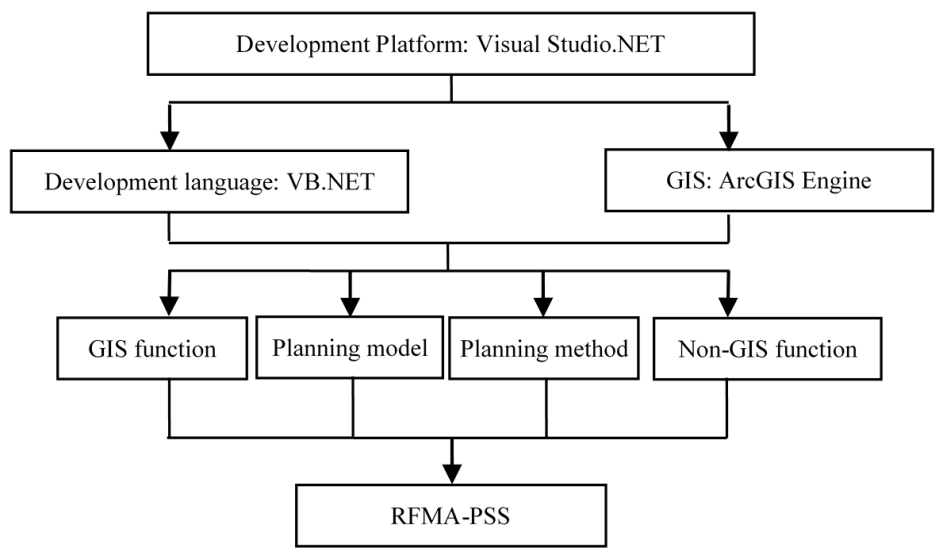

Figure 4. RMFA-PSS development platform.

\subsection{Structure of RMFA-PSS}

Generally, RMFA-PSS adopts the overall structure of "data-model and method-user interaction". It includes a database system, model base system and a human-computer interactive system (Figure 5).

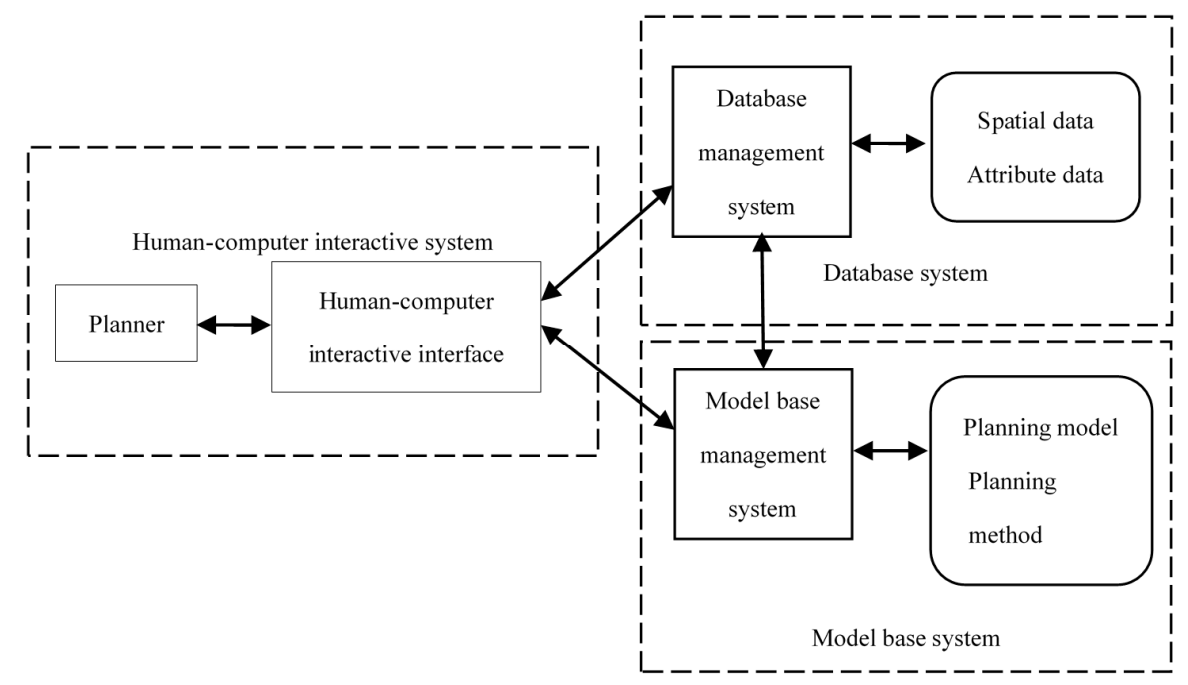

Figure 5. The overall structure of RMFA-PSS.

The database system is responsible for the construction and maintenance of database, and data retrieval, query, and statistics. It also stores various planning data including spatial data (vector data and raster data) and attribute data. The model base system is the key element which differs PSS from the general information system. It covers planning decision models and methods. The core model of RMFA-PSS is the RMFA planning decision model "carrying 
capacity-potential-pressure-resistance". The decision methods include index construction, index weight, comprehensive index, etc. In this system, decision-making method is realized by the GIS spatial analysis technology. The human-computer interaction system is composed of user and human-computer interaction interface. Its features lie in providing a variety of display and conversational modes, input and output conversional function, human-computer interaction under program control, and human-computer unification using various system components. As is the same with other software, RMFA-PSS also needs a human-computer interface to carry out interactive dialogues and operation between human and computer. In order to realize each function, it is performed under certain logic. Figure 6 shows the RMFA-PSS user interface, including menu bar, toolbar, layer information bar, map navigation window, map display window, status bar, etc.

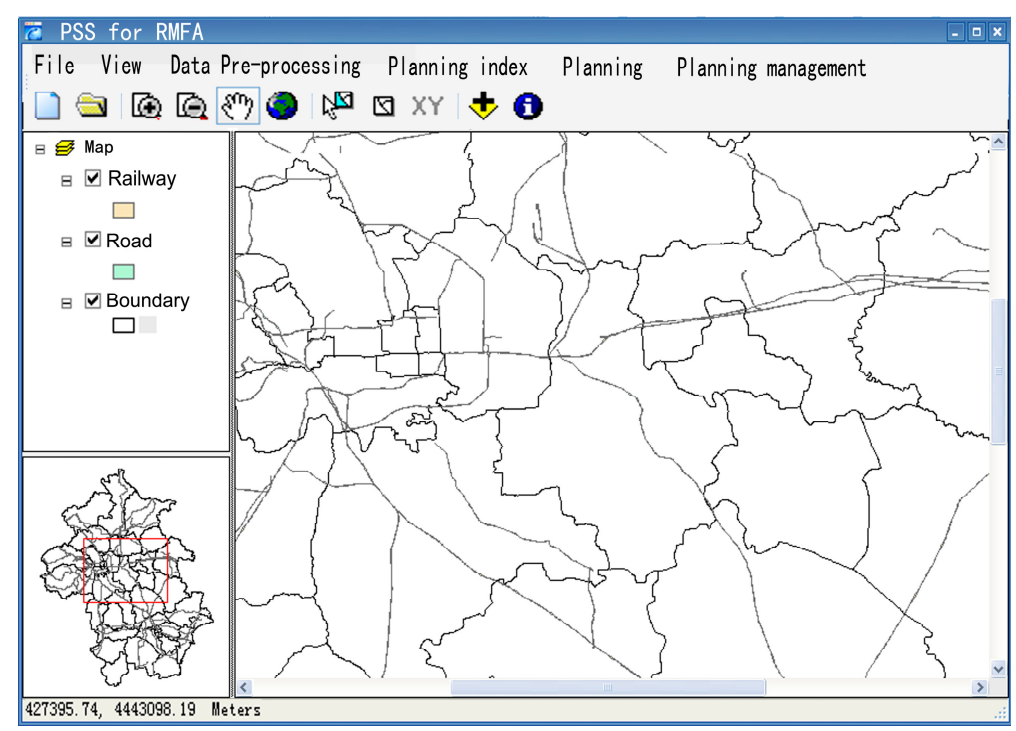

Figure 6. RMFA-PSS interface.

\subsection{Functions of RMFA-PSS}

RMFA-PSS contains numerous functional nodes. According to the properties, these nodes can be classified into five major functional modules including data management module, data pre-processing module, the construction and process of planning index module, planning module, and planning management module. These five functional modules support and connect with each other with high efficiency in planning data processing, thereby constituting an organic unified entity on the whole. Figure 7 is a diagram of the functional modules in the system.



Figure 7. The functional modules of RMFA-PSS. 


\subsection{Summary of RMFA-PSS}

The core of RMFA-PSS is the RMFA planning decision model and the decision method. It integrates the complex planning decision model solutions with classical GIS spatial analysis technology and modern decision technology organically. Moreover, RMFA-PSS highly integrates the linear weight summation decision rule with the GIS spatial overlay analysis by using the visual function of GIS. In this way, the planning decision is made based on GIS spatial analysis in the planning mode- "what you see is what you get". Thus, the planning decision process becomes simple, accurate, flexible, and efficient. The system can complete all the operations from beginning of date input to result output. If the index system, value, weight, and overlay rule are consistent with one another accordingly, the planning results can be reproducible and testable. Furthermore, this system can be used for not only RMFA planning, but also for all kinds of similar functional area planning as long as it is relevant to spatial data processing and spatial analysis either partially or wholly, such as ecological functional area planning, environmental functional area planning, and urban functional area planning. Meanwhile, if the corresponding functional area planning models and methods can be integrated into the system, the corresponding planning decision tasks will be completed as well. This feature illustrates the openness and commonality in system development, serving as reference for other types of PSS development.

\section{Application of RMFA-PSS}

\subsection{Study Area and Data}

The Beijing-Tianjin region in China was chosen to be the research area of RMFA-PSS. The area includes Beijing, Tianjin, and Langfang, covering 35,000 $\mathrm{km}^{2}$. As the economic center of the Beijing-Tianjin-Hebei area and one of the three metropolitan areas in China, the sample area is industry-intensive and densely populated, which has become a hot research spot. The data mainly focuses on the Beijing-Tianjin region, including the Landsat-7 ETM image of the study area shot on May 2011 with a spatial resolution of $30 \mathrm{~m}$, topographic maps, land use maps, relevant economic and social statistical data, etc. The land use data of the Beijing-Tianjin area in 2011 was interpreted by correcting, combining, stitching, cutting, and other processing based on the remote sensing images referring to 1:100,000 topographic maps.

\subsection{RMFA-PSS Operation}

\subsubsection{Index System}

Index data is the input of PSS. Firstly, the index choice must meet the interests and needs of administrative management, that is, administrative efficiency can be reflected in the selected indexes. Secondly, the index choice must be consistent with China's current data statistical system, to obtain the research data.

According to China's RMFA planning requirements, the four-level index system of Beijing-Tianjin area RMFA planning was established (Table 2). In the index system, the carrying capacity represents the supply capacity of regional resources and energy, and the potential represents the regional economic and social development level. The greater the sum of these two types of index values, the more natural resources and human capital will be invested in the regional spatial development. Thus, it will be more favorable for the regional spatial development, which can play a positive role in promoting regional spatial development. Pressure represents load brought by the existing regional space development against the regional environment, and ecological resistance represents the suitability degree of the regional natural ecological environment on spatial development. The greater the sum of these two types of index values, the higher is the cost that needs to be paid in order to overcome various obstacles to the spatial development which is brought by the natural environment. Therefore, it will be not conducive to the regional spatial development and play a negative role in the regional spatial development. 
Table 2. The index system of the main functional areas planning in the Beijing-Tianjin area.

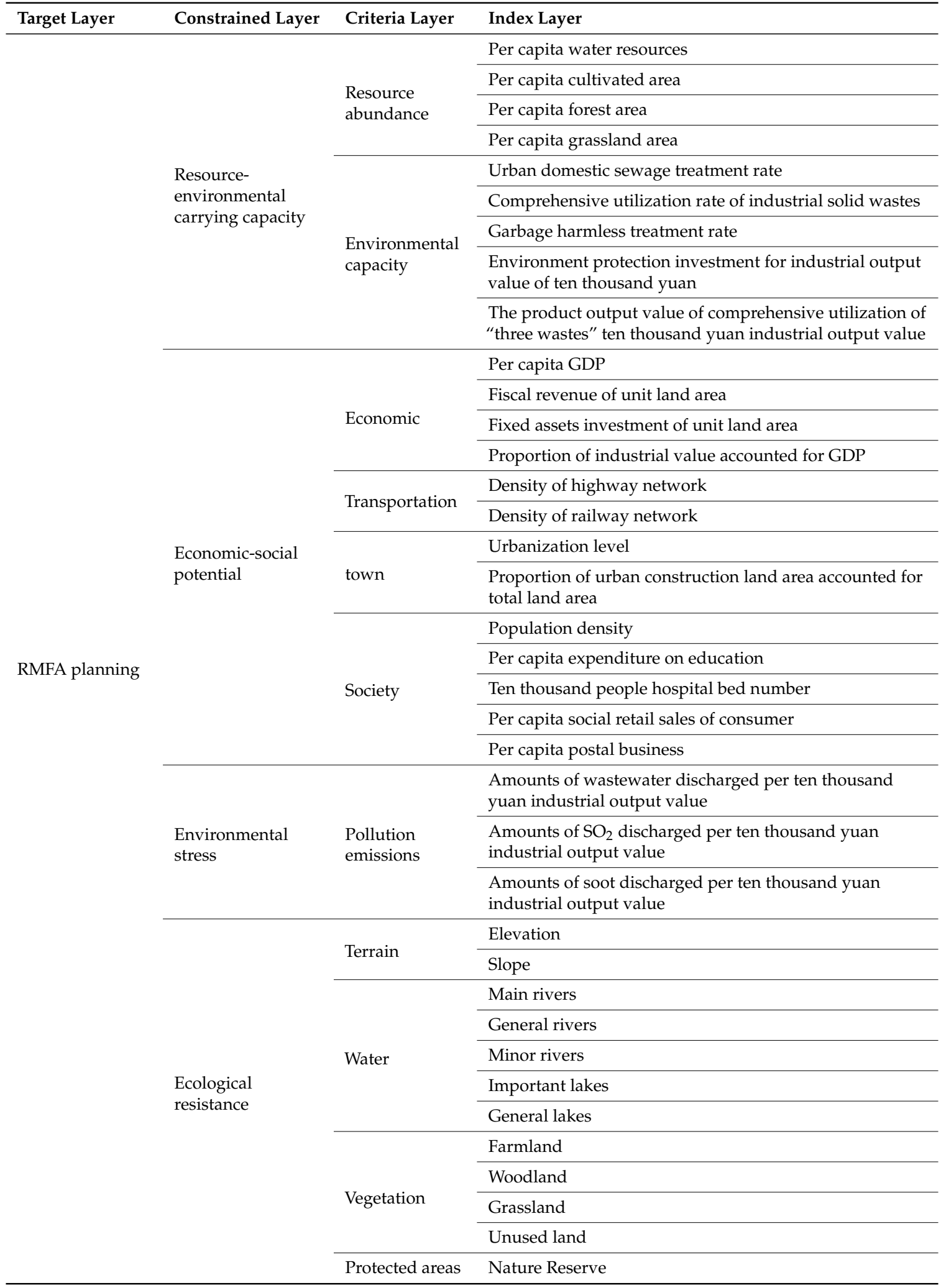

Firstly, the designed planning index system in Table 2 needs to be converted into an electronic index system in a readable XML format. This can be realized through the "construct index" command in the "planning index" menu. The interface after operation is shown in Figure 8. The electronic index system building aims to provide a template for comprehensive overlay analysis. That is to 
say, corresponding index data loaded in the system view can be chosen according to the name of each index.

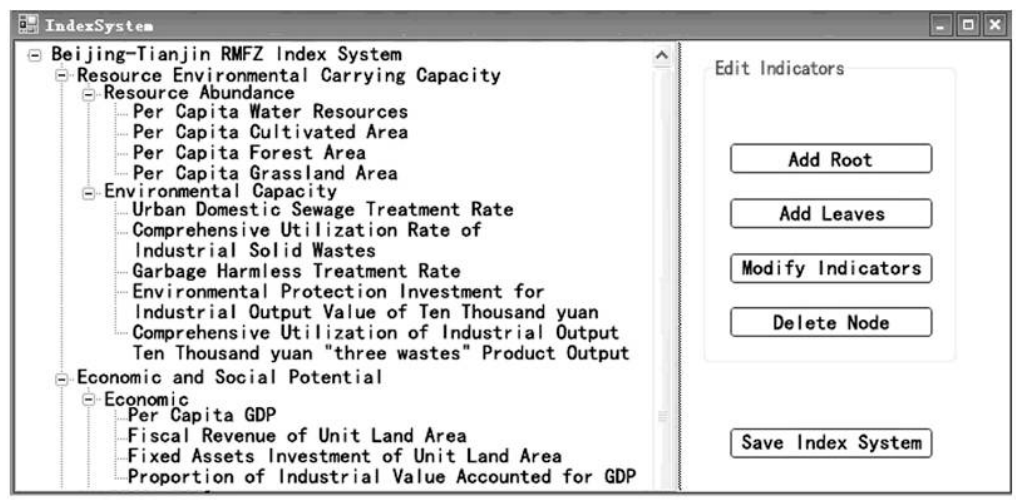

Figure 8. The interface of the planning index system of RMFA-PSS.

\subsubsection{Force Calculation}

According to formulas (3) and (4) of the RMFA planning decision model, the four forces were calculated respectively. Here, the resource-environmental carrying capacity calculation is used as an example to explain the operational process of RMFA-PSS.

(1) Index rasterization. By using "index rasterization" command in "planning index" menu, the nine resource-environmental carrying capacity indexes are converted into the rasterized data in IMG format and saved respectively. The resolution set is $30 \mathrm{~m}$ in order to be consistent with the resolution of ETM images of the study area.

(2) Index overlay synthesis. The "index synthesis" command in "planning" menu can be the completed index data loading, the index weight calculation, the index overlay analysis, and a series of other decision-making analyses (Figure 9).

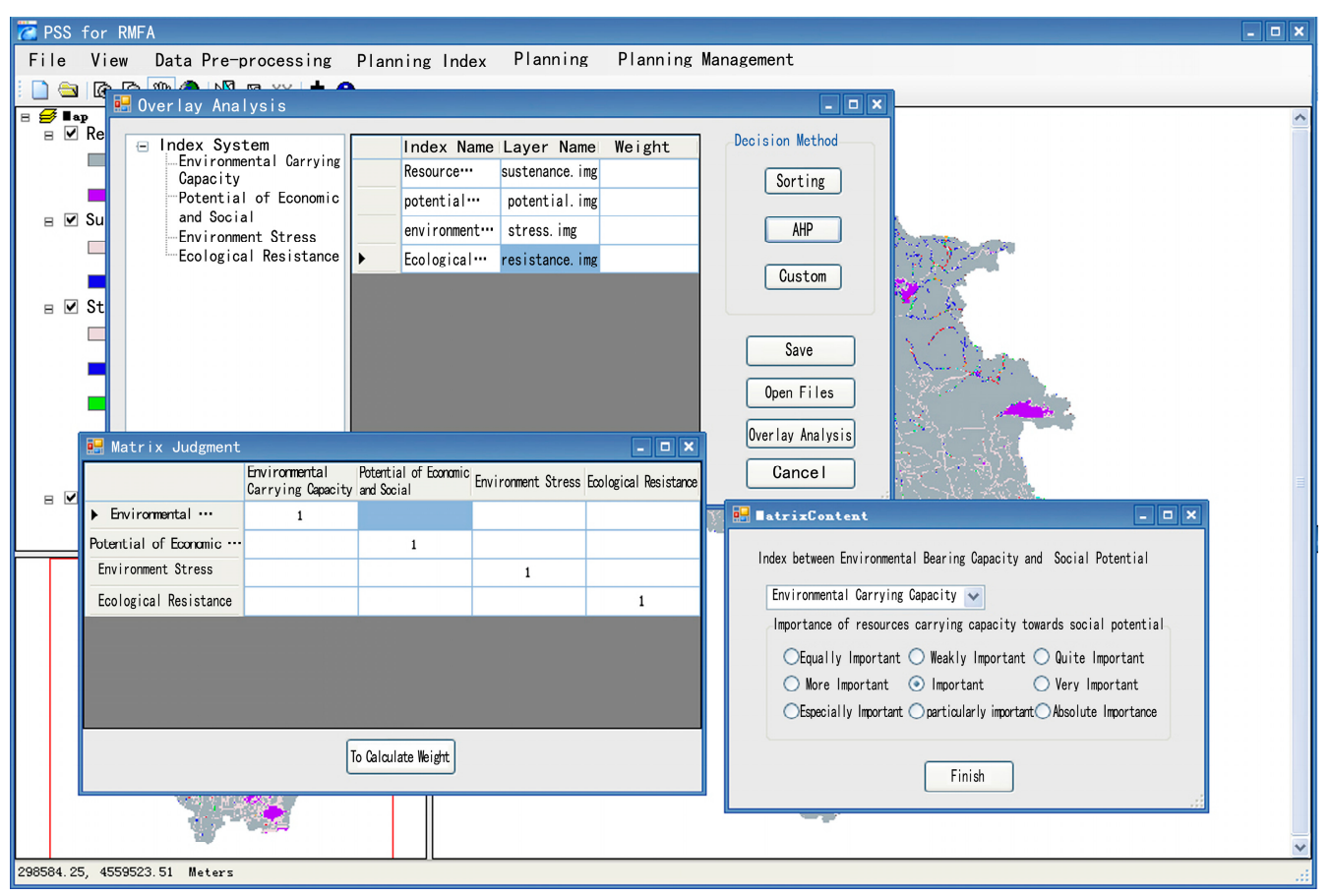

Figure 9. The "index synthesis" command interface of RMFA-PSS. 
According to Figure 2, the system integrates three kinds of index weight calculation methods: ranking method, AHP method, and the user-defined method. The ranking method and AHP method are common index weight calculation methods, while the user-defined method acts as an interface, allowing users to input the value of the index weight which is calculated by other methods. This reflects the flexibility in system development. Firstly, load the four index data concerning resource abundance into the current view in the system, and use the AHP method to calculate the weights of the four indexes. Then, click the "overlay analysis" button, and carry out spatial overlay analysis in accordance with formula (2). In this way, the rasterized data layer in the IMG format of the resource abundance index is acquired. Following a similar procedure, the rasterized data layer in IMG format of the environmental capacity index can be also calculated. Finally, the rasterized data layer in the IMG format of the resource-environmental carrying capacity is calculated by synthesizing and analyzing the resource abundance index and environmental capacity index. The weight of resource abundance index and environmental capacity index is "user-defined" by inputting 0.5 respectively. The system, in turn, facilitates the calculation of the economic-social development potential, environmental stress, and ecological resistance in the Beijing-Tianjin area, and then the rasterized data layer in the IMG format of the corresponding acting force can be completed.

\subsubsection{Comparative Analysis of Scenario Planning}

According to the characteristics of RMFA, there are two kinds of scenario planning modes namely restrictive development mode (with protection playing the predominant role and development playing the auxiliary role) and promotive development mode (with protection playing the auxiliary role and development playing the predominant role). Restrictive development mode aims to protect the ecological environment effectively during spatial development, while the promotive development mode aims to promote spatial development during rapid urbanization. As for a specific area, adopting restrictive mode or promotive mode should be determined according to the different development environments. When one mode is mainly used, the other mode becomes an alternative. For example, when the restrictive mode is mainly used, the planning scheme under restrictive mode must be preferred as the main decision-making result and will play a predominant role in spatial development, while the planning scheme under promotive mode will become the alternative and play an auxiliary role, and vice versa.

First of all, the AHP method function of the system is utilized to calculate the weights of the four fundamental forces under the two scenarios (Table 3). In the restrictive development mode, the ecological protection is defined as the main task, and therefore the weight of ecological resistance is the maximum. While in the promotive development mode, the economic and social development is the most important factor influencing decision-making, and therefore the weight of economic-social potential is the maximum.

Table 3. The weights of four forces under the two planning scenarios.

\begin{tabular}{ccc}
\hline Forces & Weights of the Restrictive Mode & Weights of the Promotive Mode \\
\hline Carrying capacity & 0.10 & 0.10 \\
Potential & 0.31 & 0.53 \\
Stress & 0.06 & 0.06 \\
Resistance & 0.53 & 0.31 \\
\hline
\end{tabular}

Secondly, overlay and synthesize the resource-environmental carrying capacity and economic-social potential in order to get the total potential; then overlay and synthesize the environmental stress and ecological resistance in order to get the total resistance. Finally, choose the "functional area planning" command in the "planning" menu (Figure 10), click the "factors synthetic" button, and execute the subtraction operation between total potential and total resistance. In this way, IPI values are evaluated. The minimum value of IPI is -0.4358 , and the maximum value 
of IPI is 0.3282 . Afterwards, click the "interval show" button, the system will regard point 0 as the cut-off point and list the IPI interval division scheme. The point 0 is fixed, while the cut-off point of point $A$ and $B$ is their median value. Point $A$ is an intermediate value between -0.4358 and 0 , point $B$ is an intermediate value between 0 and 0.3282 . Finally, click "zoning" button and four types of main functional area will be completed in terms of the three demarcation points: A, B, and 0 . The result will be displayed in the system view. Thus, under the restrictive development mode, the main functional zoning scheme of the Beijing-Tianjin area is accomplished in RMFA-PSS (Figure 11). Similarly, the zoning scheme of the promotive development mode is accomplished in RMFA-PSS (Figure 12).

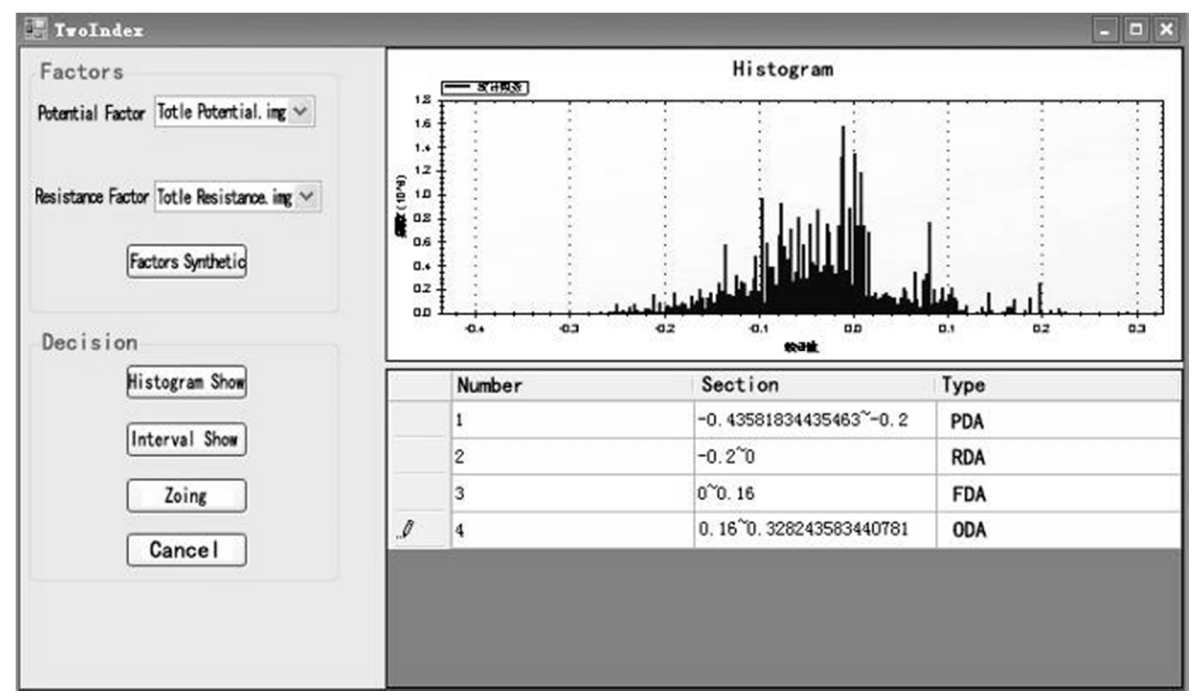

Figure 10. Functional area planning operation interface of RMFA-PSS.

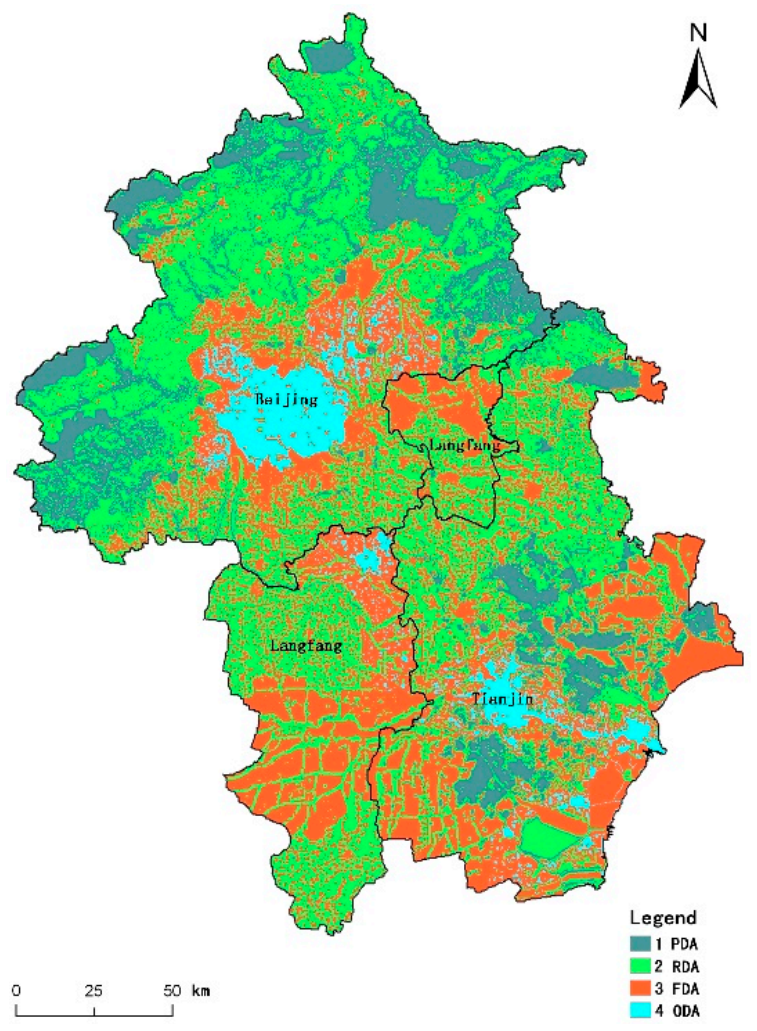

Figure 11. Result of restrictive development mode. 


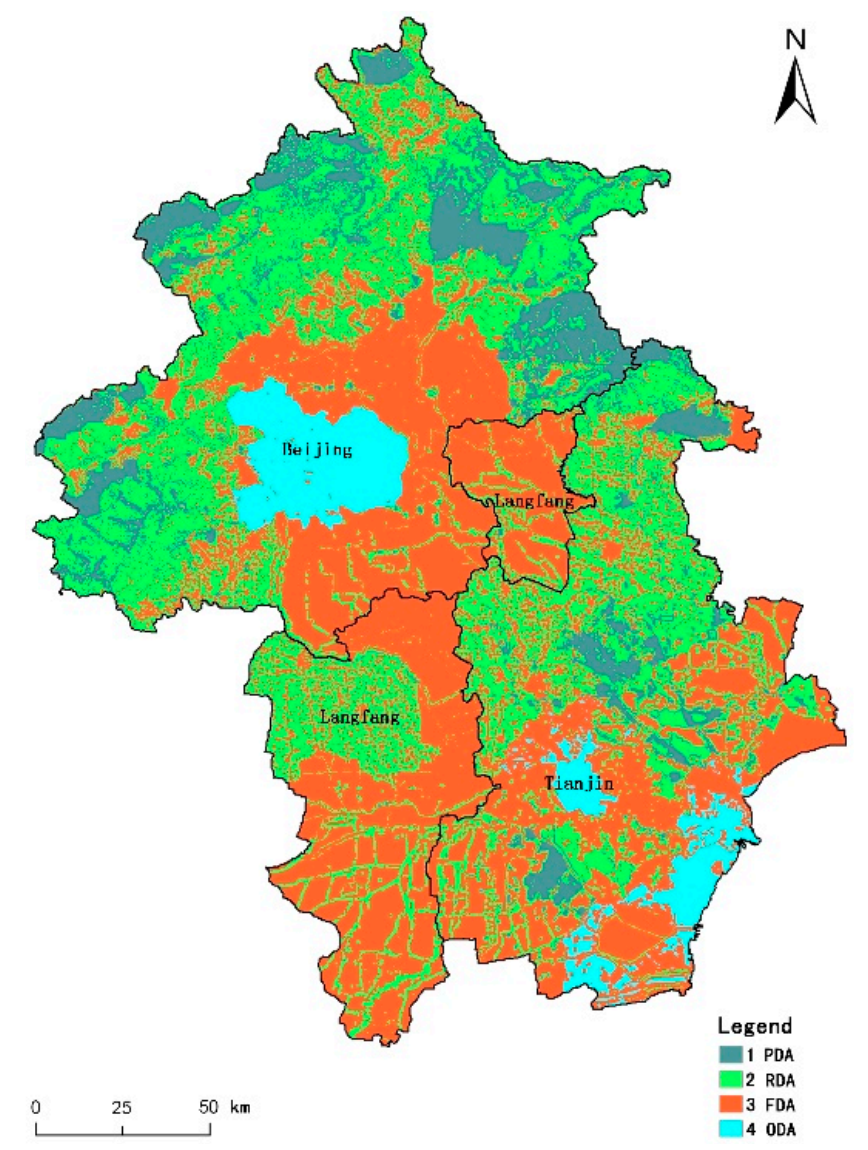

Figure 12. Result of promotive development mode.

In general, the entire Beijing-Tianjin area can be divided into four functional zones in the restrictive scenario planning scheme. From north to south, in turn, they are the ecological protection zone along the Yanshan Mountains, Beijing and its suburban development zone, Beijing-Tianjin ecologically limited zone, and the south development zone constituted by Tianjin and its suburban areas, Langfang, Bazhou, and Wenan. In the promotive scenario planning scheme, the center downtown of Beijing and Tianjin is taken as the priority concerning the development functional areas, forming a spatial pattern of "two points and one ribbon" with optimized and focus development; while restricted development zones and prohibited development zones constitute the protective and limited spatial development area surrounding Beijing and facing Tianjin.

Statistics (Table 4) show that compared with the predominant protection planning scheme, in the predominant development planning scheme, prohibited development zones account for $16.57 \%$ and reduce by $5.96 \%$; restricted development zones decline by $6.57 \%$; focus development zones increase by $10.91 \%$; optimized development zone increase $1.62 \%$. The results show, according to different development scenario analyses, better planning decisions for the Beijing-Tianjin main functional areas with multi-objectives and multi-situational constraints which can be realized by setting up the relevant weights of the four fundamental forces in the planning model.

RMFA-PSS is utilized to give two different planning schemes by changing the weights of the four forces to change the decision making rules. According to the above analysis for PSS, it can clearly be seen that PSS does not choose the best solution among all, the final choice of scheme and the subsequent planning strategies of the study area should be carried out by the planners. 
Table 4. The area and proportion of each functional area in the Beijing-Tianjin area under two scenarios.

\begin{tabular}{ccccc}
\hline \multirow{2}{*}{ Functional Area Type } & \multicolumn{2}{c}{ Restrictive Development Mode } & \multicolumn{2}{c}{ Promotive Development Mode } \\
\cline { 2 - 5 } & Area $\mathbf{( k m}^{\mathbf{2}} \mathbf{)}$ & Proportion (\%) & Area $\left.\mathbf{( k m}^{\mathbf{2}}\right)$ & Proportion $\mathbf{( \% )}$ \\
\hline PDA & 7752.06 & 22.53 & 5701.36 & 16.57 \\
RDA & 15927.33 & 46.29 & 13666.75 & 39.72 \\
FDA & 8997.62 & 26.15 & 12751.50 & 37.06 \\
ODA & 1730.71 & 5.03 & 2288.11 & 6.65 \\
\hline
\end{tabular}

Firstly, the actual development environment of the Beijing-Tianjin area is analyzed. As one of the key development areas at national level, in building an international metropolis region of strategic context, driven by Beijing and Tianjin, both cities at the national-center level, the urbanization and the rapid development of the Beijing-Tianjin area is inevitable. Therefore, the main functional area planning scheme under the two different scenarios in the guide, in this promotive development mode with protection playing the auxiliary role and development playing the predominant role is more consistent with the actual situation in the Beijing-Tianjin area, which is more in line with the requirements of future development strategy in Beijing and Tianjin. This should be the primary and preferable option, and restrictive mode should be the alternative option.

Secondly, in full consideration of the natural environment constraints and limitations in the Beijing-Tianjin area in the future, the main goal of the spatial development in the Beijing-Tianjin area is to achieve rapid economic and social development, as well as the orderly expansion of the urbanized area. The strategic direction of spatial development is as follows; first, Beijing extends to Baoding City which is located in the southwest; second, it develops to the south along the Beijing City-Tianjin City-Tanggu City axis; third, it expands to Langfang City in the east. At the same time, Tianjin City should extend to the northern and western areas. Beijing and Tianjin will be expanding themselves into two international central cities in China.

\section{Discussion and Conclusions}

This paper introduces advanced PSS to the field of regional spatial functional zoning by explaining the basic concept, characteristics, and principles of PSS. At the same time, the paper takes the PSS development for the latest planning of RMFA in China as an example. The RMFA-PSS has been established on the basis of the component-type secondary development technology of GIS. The research can provide references for development and application of PSS for other types of spatial functional zoning. Specifically, on the Visual Basic .NET visualization development platform, the planning decision model, planning decision method, and GIS are organically integrated and customized by using the ArcGIS Engine as the component-type secondary development technology and VB.NET as the development language. Therefore, RMFA-PSS is established tailored to the regional main functional areas of planning. Such RMFA-PSS was applied to the planning of the Beijing-Tianjin area, and the result conforms to the objective reality of this area development. That is to say, the system is preferably applicable. This illustrates that RMFA-PSS is a systemic and integrated operating entity with a tight structure. It integrates the planning decision model and the planning decision method, and could in turn provide better decision support for the regional main functional areas of planning in utility and flexibility.

This study showed that: firstly, PSS can improve the efficiency and effectiveness of planning, it is able to offer a number of schemes under various scenarios, and at the same time planners must complete the final scheme selection and the subsequent development strategies formulation. Secondly, the planning decision rule is the premise and basis of PSS, PSS is essentially a multi-scheme comparison technology platform based on different decision rules. The decision rules are mainly shown as the planning model and decision making methods in RMFA-PSS. Thirdly, during the application of RFMA-PSS in the Beijing-Tianjin area, the decision rules design for planning, embodied in the 
index weights, change, by changing the weights of the forces to obtain the planning schemes under the two scenarios, and then bring forward the future development strategy according to the final planning scheme.

Although, in this paper, RMFA-PSS system was established under the integrated idea of the decision model, decision method, and GIS, it actually integrated only a part of the GIS function concerning data processing and spatial analysis. Compared with the commercial GIS software, the RMFA-PSS system is less powerful and more complex. For example, there are still some basic data processing functions, which need support from the traditional commercial GIS software and other software. Therefore, it is necessary to keep up the in-depth study in system designing. However, as a secondary development support system, its main purpose is to solve the problems in regional main functional areas planning. If these goals are reached, then the system would be considered to have achieved the expected development target. Additionally, the existing shortcomings can provide motivation and theoretical foundation for further advancement and upgrade of the RMFA-PSS.

Acknowledgments: Great thanks are given to the National Natural Science Foundation of China (41201168) and the Fundamental Research Funds for the Central Universities of China (2013HGXJ0207).

Author Contributions: Xiaorui Zhang conceived and designed the research; Xiaorui Zhang and Qian Hua analyzed the data and wrote the paper; Qian Hua performed the data collection; Linya Zhang analyzed some data and checked the language of the manuscript.

Conflicts of Interest: The authors declare no conflict of interest.

\section{References}

1. Xie, G.D.; Lu, C.X.; Zhen, L. Objective, progress and methodology of spatial function zoning. Geogr. Res. 2009, 28, 561-570.

2. Huo, B. Revival and novation of strategic spatial planning of China. City Plan. Rev. 2007, 31, 19-29.

3. Zhang, X.R.; Fang, C.L.; Wang, Z.B. Urban construction land suitability evaluation based on improved multi-criteria evaluation based on GIS (MCE-GIS): Case of New Hefei City, China. Chin. Geogr. Sci. 2013, 23, 740-753. [CrossRef]

4. Stillwell, J. Planning Support Systems in Practice (Advances in Spatial Science); Springer: Heidelberg, Germany, 2002.

5. Harris, B. Beyond geographic information systems: Computers and the planning professional. J. Am. Plan. Assoc. 1989, 55, 85-90. [CrossRef]

6. Geertman, S.; Toppen, F.; Stillwell, J. Planning Support Systems for Sustainable Urban Development; Springer: Heidelberg, Germany, 2013.

7. Landis, L.D. Imagining land use futures: Applying the California Urban Future Model. J. Am. Plan. Assoc. 1995, 61, 438-457. [CrossRef]

8. Clarke, K.C.; Gaydos, L.; Hoppen, S. A self-modifying cellular automaton model of historical urbanization in the San Francisco Bay area. Environ. Plan. B Plan. Des. 1997, 24, 247-261. [CrossRef]

9. Demetriou, D.; Stillwell, J.; See, L. Land consolidation in Cyprus: Why is an integrated planning and decision support system required? Land Use Policy 2012, 29, 131-142. [CrossRef]

10. Kocabas, V.; Dragicevic, S.; Mccann, E. Integration of a GIS-Bayesian network agent-based model in a planning support system as framework for policy generation. J. Urban Reg. Inf. Syst. Assoc. 2012, 24, 35-52.

11. Pettit, C.J. Use of a collaborative GIS-based planning-support system to assist in formulating a sustainable-development scenario for Hervey Bay, Australia. Environ. Plan. B Plan. Des. 2005, 32, 523-545. [CrossRef]

12. Katoshevski, C.R.; Arentze, T.; Timmermans, H. A multi-agent planning support-system for assessing externalities of urban form scenarios: Results of case studies. Sustainability 2010, 2, 2253-2278. [CrossRef]

13. Coutinho, R.J.; Simao, A.; Antunes, C.H. A GIS-based multicriteria spatial decision support system for planning urban infrastructures. Decis. Support Syst. 2011, 51, 720-726. [CrossRef]

14. Kang, M.H.; Choi, H.R.; Kim, H.S. Development of a maritime transportation planning support system for car carriers based on genetic algorithm. Appl. Intell. 2012, 36, 585-604. [CrossRef] 
15. Long, Y.; Mao, Q.Z. Urban planning support system definition, objectives and framework. J. Tsinghua Univ. Sci. Technol. 2010, 50, 335-337.

16. Du, N.R.; Li, Y. Planning support system (PSS) and its application to decision-making for urban spatial development. Eng. J. Wuhan Univ. 2005, 38, 137-142.

17. Yeh, A.G.; Song, X.D.; Niu, X.Y. Geographic Information and Planning Support System; Science Press: Beijing, China, 2006.

18. Niu, X.Y. Planning Support System: A new approach for computer-aided planning. Urban Plan. Forum 2006, 2, 96-101.

19. Zhao, Y.; Shang, J.C.; Xu, L. Establishment and application of urbanization strategic environmental assessment integration technology system. Chin. Geogr. Sci. 2008, 18, 184-192. [CrossRef]

20. Yang, L.; Zhang, Y.F.; Wang, B.C. Soil utilization and planning support system of the villages and towns based on GIS. Xinjiang Agric. Sci. 2008, 45, 264-269.

21. Long, Y.; Shen, Z.J.; Mao, Q.Z. Urban growth control planning support system: approach, implementation and application. City Plan. Rev. 2011, 35, 62-71.

22. Zhang, Y.; Zhang, H.Q.; Ni, D.Y. Agricultural land use optimal allocation system in developing area: Application to Yili watershed, Xinjiang Region. Chin. Geogr. Sci. 2012, 2, 232-244. [CrossRef]

23. Fan, J.; Sun, W.; Zhou, K. Major function oriented zone: New method of spatial regulation for reshaping regional development pattern in China. Chin. Geogr. Sci. 2012, 22, 196-209. [CrossRef]

24. Zhang, X.R.; Zong, Y.G. Application of model and method in the planning of region major functional zones-A case study of Beijing-Tianjin Region, China. Sci. Geog. Sin. 2010, 30, 728-734.

25. Tang, G.A.; Yang, X. ArcGIS Spatial Analysis Experiments Tutorial; Science Press: Beijing, China, 2006.

(C) 2016 by the authors; licensee MDPI, Basel, Switzerland. This article is an open access article distributed under the terms and conditions of the Creative Commons Attribution (CC-BY) license (http:/ / creativecommons.org/licenses/by/4.0/). 